\title{
819.
}

\section{ON TWO CASES OF THE QUADRIC TRANSFORMATION BETWEEN TWO PLANES.}

[From the Johns Hopkins University Circulars, No. 13 (1882), pp. 178, 179.]

SEeking for the coordinates $x_{3}, y_{3}, z_{3}$ of the third point of intersection of the cubic curve $x^{3}+y^{3}+z^{3}+6 l x y z=0$ by the line through any two points $\left(x_{1}, y_{1}, z_{1}\right)$, $\left(x_{2}, y_{2}, z_{2}\right)$ on the curve, the expressions present themselves in the form

where

$$
x_{3}: y_{3}: z_{3}=P+2 l A: Q+2 l B: R+2 l C,
$$

$$
\begin{array}{ll}
P=x_{1} y_{1} y_{2}{ }^{2}+z_{1} x_{1} z_{2}{ }^{2}-y_{1}{ }^{2} x_{2} y_{2}-z_{1}{ }^{2} z_{2} x_{2}, & A=x_{1}{ }^{2} y_{2} z_{2}-y_{1} z_{1} x_{2}{ }^{2}, \\
Q=y_{1} z_{1} z_{2}{ }^{2}+x_{1} y_{1} x_{2}{ }^{2}-z_{1}^{2} y_{2} z_{2}-x_{1}{ }^{2} x_{2} y_{2}, & B=y_{1}{ }^{2} z_{2} x_{2}-z_{1} x_{1} y_{2}{ }^{2}, \\
R=z_{1} x_{1} x_{2}{ }^{2}+y_{1} z_{1} y_{2}{ }^{2}-x_{1}{ }^{2} z_{2} x_{2}-y_{1}{ }^{2} y_{2} z_{2}, & C=z_{1}{ }^{2} x_{2} y_{2}-x_{1} y_{1} z_{2}{ }^{2}
\end{array}
$$

but it is known that, in virtue of

$$
U_{1}=x_{1}^{3}+y_{1}^{3}+z_{1}^{3}+6 l x_{1} y_{1} z_{1}=0, \quad U_{2}=x_{2}^{3}+y_{2}^{3}+z_{2}^{3}+6 l x_{2} y_{2} z_{2}=0,
$$

which connect the coordinates $\left(x_{1}, y_{1}, z_{1}\right)$ and $\left(x_{2}, y_{2}, z_{2}\right)$, we have $P: Q: R=A: B: C^{*}$, so that the coordinates $\left(x_{3}, y_{3}, z_{3}\right)$ of the third point of intersection may be expressed indifferently in the two forms

$$
x_{3}: y_{3}: z_{3}=P: Q: R \text {, and } x_{3}: y_{3}: z_{3}=A: B: C .
$$

But these considered irrespectively of the equations $U_{1}=0, U_{2}=0$, are distinct formulæ, each of them separately establishing a correspondence between the three points $\left(x_{1}, y_{1}, z_{1}\right),\left(x_{2}, y_{2}, z_{2}\right),\left(x_{3}, y_{3}, z_{3}\right)$, or if we regard one of these points as a fixed point, then a correspondence between the remaining two points, or if we consider these as belonging each to its own plane, then a correspondence between two planes.

* See Sylvester on Rational Derivation of Points on Cubic Curves, Amer. Jour. of Math. vol. III. p. 62. 
Writing for convenience $(a, b, c)$ for the coordinates of the fixed point, and $\left(x_{1}, y_{1}, z_{1}\right)$, $\left(x_{2}, y_{2}, z_{2}\right)$ for those of the other two points, the formulæ with $A, B, C$ give thus the correspondence

$$
x_{2}: y_{2}: z_{2}=b c x_{1}^{2}-a^{2} y_{1} z_{1}: c a y_{1}^{2}-b^{2} z_{1} x_{1}: a b z_{1}^{2}-c^{2} x_{1} y_{1}
$$

which is the first of the two cases in question. These equations give reciprocally

$$
x_{1}: y_{1}: z_{1}=b c x_{2}^{2}-a^{2} y_{2} z_{2}: c a y_{2}{ }^{2}-b^{2} z_{2} x_{2}: a b z_{2}{ }^{2}-c^{2} x_{2} y_{2},
$$

or the correspondence is a $(1,1)$ quadric correspondence.

The formulæ with $P, Q, R$ give in like manner

$$
x_{2}: y_{2}: z_{2}=a\left(a x_{1}^{2}+b y_{1}^{2}+c z_{1}^{2}\right)-x_{1}\left(a^{2} x_{1}+b^{2} y_{1}+c^{2} z_{1}\right), \quad \& c .,
$$

or if for shortness

$$
\Omega_{1}=a x_{1}^{2}+b y_{1}^{2}+c z_{1}^{2}, \quad \Theta_{1}=a^{2} x_{1}+b^{2} y_{1}+c^{2} z_{1},
$$

then

$$
x_{2}: y_{2}: z_{2}=a \Omega_{1}-x_{1} \Theta_{1}: b \Omega_{1}-y_{1} \Theta_{1}: c \Omega_{1}-z_{1} \Theta_{1},
$$

which is the second of the two cases. We have reciprocally

$$
x_{1}: y_{1}: z_{1}=a \Omega_{2}-x_{2} \Theta_{2}: b \Omega_{2}-y_{2} \Theta_{2}: c \Omega_{2}-z_{2} \Theta_{2},
$$

where

$$
\Omega_{2}=a x_{2}{ }^{2}+b y_{2}{ }^{2}+c z_{2}{ }^{2}, \quad \Theta_{2}=a^{2} x_{2}+b^{2} y_{2}+c^{2} z_{2},
$$

and the correspondence is thus in this case also a $(1,1)$ quadric correspondence. 